\title{
Predicting Prolonged Length of Hospital Stay for Peritoneal Dialysis-Treated Patients Using Stacked Generalization: Model Development and Validation Study
}

Guilan Kong ${ }^{1,2^{*}}, \mathrm{PhD}^{\ddagger}$; Jingyi $\mathrm{Wu}^{2^{*}}$, MS; Hong $\mathrm{Chu}^{3}$, MD, PhD; Chao Yang ${ }^{3}$, MS; Yu Lin ${ }^{4}$, MPH; Ke Lin ${ }^{1}$, MS; Ying $\mathrm{Shi}^{5}$, BMS; Haibo Wang ${ }^{1,6}$, MSc, MPH, MBBS; Luxia Zhang ${ }^{1,2,3}$, MPH, MD

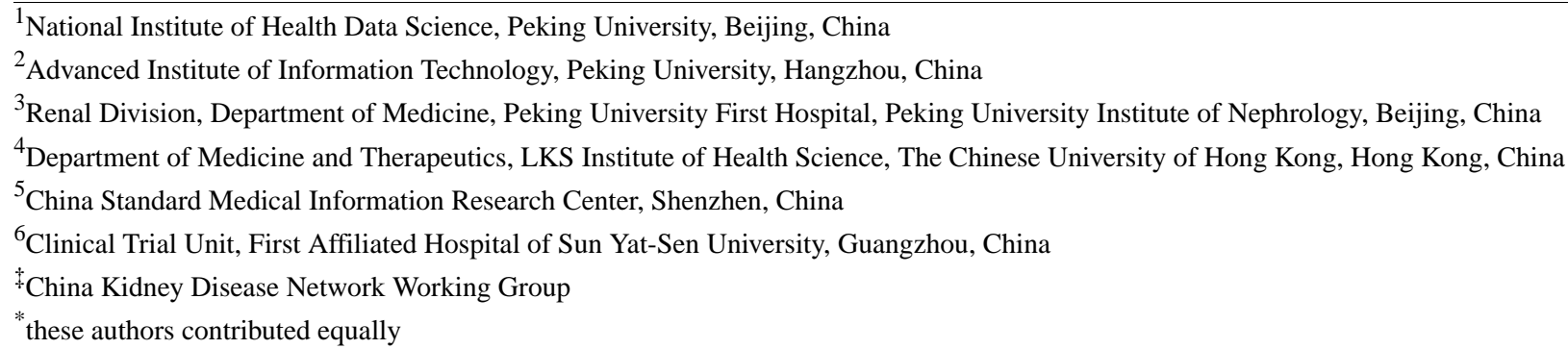

\section{Corresponding Author:}

Luxia Zhang, MPH, MD

National Institute of Health Data Science

Peking University

No 38 Xueyuan Road

Haidian District

Beijing, 100191

China

Phone: 861082806538

Email: zhanglx@bjmu.edu.cn

\section{Abstract}

Background: The increasing number of patients treated with peritoneal dialysis (PD) and their consistently high rate of hospital admissions have placed a large burden on the health care system. Early clinical interventions and optimal management of patients at a high risk of prolonged length of stay (pLOS) may help improve the medical efficiency and prognosis of PD-treated patients. If timely clinical interventions are not provided, patients at a high risk of pLOS may face a poor prognosis and high medical expenses, which will also be a burden on hospitals. Therefore, physicians need an effective pLOS prediction model for PD-treated patients.

Objective: This study aimed to develop an optimal data-driven model for predicting the pLOS risk of PD-treated patients using basic admission data.

Methods: Patient data collected using the Hospital Quality Monitoring System (HQMS) in China were used to develop pLOS prediction models. A stacking model was constructed with support vector machine, random forest (RF), and K-nearest neighbor algorithms as its base models and traditional logistic regression (LR) as its meta-model. The meta-model used the outputs of all 3 base models as input and generated the output of the stacking model. Another LR-based pLOS prediction model was built as the benchmark model. The prediction performance of the stacking model was compared with that of its base models and the benchmark model. Five-fold cross-validation was employed to develop and validate the models. Performance measures included the Brier score, area under the receiver operating characteristic curve (AUROC), estimated calibration index (ECI), accuracy, sensitivity, specificity, and geometric mean $(\mathrm{Gm})$. In addition, a calibration plot was employed to visually demonstrate the calibration power of each model.

Results: The final cohort extracted from the HQMS database consisted of 23,992 eligible PD-treated patients, among whom $30.3 \%$ had a pLOS (ie, longer than the average LOS, which was 16 days in our study). Among the models, the stacking model achieved the best calibration (ECI 8.691), balanced accuracy (Gm 0.690), accuracy (0.695), and specificity (0.701). Meanwhile, the stacking and RF models had the best overall performance (Brier score 0.174 for both) and discrimination (AUROC 0.757 for 
the stacking model and 0.756 for the RF model). Compared with the benchmark LR model, the stacking model was superior in all performance measures except sensitivity, but there was no significant difference in sensitivity between the 2 models. The 2-sided $t$ tests revealed significant performance differences between the stacking and LR models in overall performance, discrimination, calibration, balanced accuracy, and accuracy.

Conclusions: This study is the first to develop data-driven pLOS prediction models for PD-treated patients using basic admission data from a national database. The results indicate the feasibility of utilizing a stacking-based pLOS prediction model for PD-treated patients. The pLOS prediction tools developed in this study have the potential to assist clinicians in identifying patients at a high risk of pLOS and to allocate resources optimally for PD-treated patients.

(JMIR Med Inform 2021;9(5):e17886) doi: $\underline{10.2196 / 17886}$

\section{KEYWORDS}

peritoneal dialysis; prolonged length of stay; machine learning; prediction model; clinical decision support

\section{Introduction}

Over the past 30 years, the United States Renal Data System has reported a rapid increase in the incidence of end-stage kidney disease (ESKD) [1]. The increasing number of patients with ESKD treated with kidney replacement therapy_including hemodialysis, peritoneal dialysis (PD), and renal transplantation-has put a large burden on the health care system. Approximately 2.6 million people worldwide received kidney replacement therapy in 2010 [2], and the prevalence of ESKD in China was 237.3 cases per million population in 2012 [3]. In 2015, the average inpatient expenditure for patients with ESKD in China was approximately $¥ 24,800$ (US \$3793) [4], and the total inpatient expenditure for patients with ESKD in China was in excess of $¥ 6.75$ billion (US $\$ 1.03$ billion). In 2016 , the average expenditure on patients with ESKD in the United States was estimated to be US $\$ 50$ billion, one-third of which was attributed to hospitalization costs [1]. Hospitalization remains a critical outcome for patients with ESKD, and the risk of hospitalization in patients undergoing dialysis is triple that of patients without ESKD [5]. In-hospital length of stay (LOS) is a key indicator of the efficiency of inpatient management. Prolonged LOS (pLOS) is associated not only with high resource consumption and medical expenses [6,7] but also with a high risk of complications [8]. Much attention has been given to reducing hospitalization costs [9-15], but few studies have focused on preventing pLOS for PD-treated patients. The increasing number of PD-treated patients and their consistently high hospital admission rate have placed a large burden on the health care system. An accurate pLOS prediction model can assist physicians to risk-stratify patients and optimally allocate health care resources [7,16]. Early clinical interventions and optimal management of patients at a high risk of pLOS may help reduce hospitalization expenses and improve prognosis for PD-treated patients $[7,8,17]$. If timely clinical interventions are not provided, patients at a high risk of pLOS may face poor prognosis and high medical expenses, which will also burden hospitals [18].

Given the increasing number of patients undergoing dialysis and the importance of optimal resource allocation, physicians need an effective LOS prediction model. However, no well-developed LOS prediction models for patients undergoing dialysis can be found in the literature. Some other risk-stratification models for patients undergoing dialysis use mortality [19-21] or cardiovascular events [22] as the end point. Wagner et al [20] used a nationwide, multicenter, prospective cohort study in the United Kingdom (the UK Renal Registry) as a data source to develop a Cox proportional hazards model for predicting long-term mortality in incident dialysis patients. They found that using basic patient characteristics, comorbid conditions, and laboratory variables to predict the 3-year mortality of incident dialysis patients had sufficient accuracy. Quinn et al [21] used a Canadian administrative health database to develop a prognostic index for 1-year mortality in patients undergoing dialysis by combining logistic regression (LR) with different variable selection methods. Matsubara et al [22] used data from the Japan Dialysis Outcomes and Practice Patterns Study to develop an LR model for predicting the incidence of cardiovascular events among patients undergoing hemodialysis. However, few models use LOS as the prediction outcome.

Meanwhile, a number of studies have explored the factors affecting the LOS of patients undergoing dialysis. Allon et al [23] explored the association of hospitalization outcomes with clinical factors and laboratory parameters in patients undergoing hemodialysis and found that infection-related hospitalization was associated with pLOS. Kshirsagar et al [24] compared the LOS of hemodialysis patients receiving care from nephrologists and internists and found that the LOS was significantly shorter for patients under the care of nephrologists than for patients under the care of internists. Rocco et al [25] studied the risk factors for hospitalization in patients receiving chronic dialysis and confirmed that the risk factors for LOS were similar to those for mortality. Other factors affecting the LOS of patients undergoing dialysis have also been explored, such as obesity [26], hemoglobin level [27], admission diagnosis [28], and comorbidities [23,29]. However, no study has built an effective model for pLOS prediction in patients undergoing dialysis.

With the exponential increase in the amount of health care data, machine learning algorithms have gained special attention for their capabilities of handling high-dimension and large-scale data. Some machine learning-based LOS prediction models have been developed for patients with other diseases. The prediction outcome of existing LOS prediction models could be classified into 2 types: (1) numeric LOS and (2) binary outcome (ie, having a pLOS or not). Moran et al [30] constructed a numeric LOS prediction model for patients in the intensive care unit (ICU) by using a traditional linear regression model. Their results suggested that their LOS prediction model 
performed well in predicting the average LOS of patients in the ICU but showed limited performance in predicting the LOS of individual patients. Yang et al [31] developed a numeric LOS prediction model based on the support vector machine (SVM) algorithm for burn patients at different stages and compared its prediction performance with that of the traditional linear regression model. They found that although the SVM model was more effective than the linear regression model in LOS prediction for burn patients, it yielded a high mean relative error of $43.9 \%$. LaFaro et al [32] developed a numeric LOS prediction model based on the artificial neural network (ANN) algorithm for patients in the ICU after cardiac surgery. Their results also suggested that the ANN-based LOS prediction model outperformed the traditional linear regression model $\left(R^{2}: 0.410\right.$ vs $0.200 ; \mathrm{R}^{2}$ measures the goodness of fit of the corresponding model), but the prediction performance of the ANN-based model was still limited. However, if patients are classified into 2 groups (ie, with and without pLOS), the difference in LOS patterns between patients in the 2 groups could be more obvious and easily discovered, and this classification helps identify typical LOS patterns and improve the performance of LOS prediction models [33]. In the literature, the LOS prediction models with binary outcomes achieved good performance. Ma et al [34] developed a personalized pLOS prediction model for patients in the ICU by combining just-in-time learning and one-class extreme learning machine algorithms and found that the model achieved superior performance to the traditional binary classification algorithms. Chuang et al [35] compared the performance of various supervised learning approaches with an LR model in pLOS prediction for general surgery patients and the results showed that the random forest (RF) model outperformed the LR model. Morton et al [36] used 5 machine learning algorithms to predict the pLOS of hospitalized patients with diabetes and found that the SVM model demonstrated the best prediction performance, followed closely by the RF model. However, LOS prediction models based on machine learning technologies for PD-treated patients remain to be developed.

Stacked generalization, or stacking, is a general ensemble method that combines different types of machine learning models ("base models") through an aggregation model ("meta-model") to maximize the prediction performance [37]. Several studies [38,39] have found that ensemble learning methods can produce a better or equal predictive performance than their component parts. Lertampaiporn et al [38] developed a heterogeneous ensemble model for microRNA precursor classification through a voting system. Their results showed that the ensemble method produced a more reliable prediction than its base classifiers. Wang et al [39] used the stacking algorithm to predict membrane protein types, and the ensemble model yielded a better overall performance than its base models. Phan et al [40] developed a stacking model to predict cancer survival and reported that this model outperformed the majority-vote model. An ensemble of various machine learning models could help reduce the bias in a single machine learning algorithm to provide a much better prediction performance than single models.

This study aimed to develop an optimal data-driven pLOS prediction model for PD-treated patients by using basic admission data from a national database. A pLOS prediction model was constructed for PD-treated patients by using the stacking method, and the Hospital Quality Monitoring System (HQMS) database in China was used for model development. An LR-based pLOS prediction model was built and considered as the benchmark model. The RF, SVM, and K-nearest neighbor $(\mathrm{KNN})$ algorithms were employed as the base models because of their superior performance in constructing ensemble models $[38,41]$, and the LR model was used as the meta-model for constructing the stacking model.

\section{Methods}

\section{Data Set and Subjects}

In this study, the HQMS database-a mandatory, patient-level national database in China-was used for data extraction and model development. The HQMS database is a large database consisting of standardized electronic inpatient discharge records, including 878 Class 3 hospitals in China [42]. The standardized electronic inpatient discharge record is a national standard medical record with a stringent standard format across different hospitals in China. The standardized electronic inpatient discharge records of patients must be filled in by clinicians who have the most comprehensive understanding of the patients' medical conditions to ensure their validity. Strict automated data quality control was performed on the HQMS data reporting system. The completeness, accuracy, and consistency of data were assessed at the time of data submission to the HQMS. Patient demographic characteristics, clinical diagnoses, medical procedures, pathology diagnoses, and medical expenditures were included in the HQMS database.

This study was reviewed and approved by the Ethics Committee of Peking University First Hospital (2015-928). The HQMS data set used in this study spans from 2013 to 2015.

Patient records of individuals who met the following criteria were extracted from the HQMS data set: (1) aged between 18 and 100 years, and (2) treated with PD. Exclusion criteria were as follows: (1) diagnosed with acute kidney injury or kidney transplantation, and (2) died in the hospital. For patients readmitted on the same day as hospital discharge, we recalculated their LOS by merging the back-to-back admission records. The PD-treated patients were identified through admission and discharge diagnoses or in-hospital medical operations by using the International Statistical Classification of Diseases, Tenth Revision (ICD-10) codes (Multimedia Appendix 1). For PD-treated patients with several discontinuous hospitalizations, we randomly selected one record for each patient to ensure that all observations were independent and that PD-treated patients with varying severities were included for model development.

\section{Outcome and Predictor Variables}

The prediction outcome of this study was binary (ie, having a pLOS or not). LOS was defined as the period from admission to discharge. pLOS was defined as an LOS longer than the average LOS, which is 16 days for patients with ESKD in China [43]. Patients with pLOS may have serious medical situations and thus need a longer hospital stay. We adopted this pLOS 
definition in our study by referring to existing studies [44-46] and consulting with experienced clinicians. The pLOS prediction models developed in our study aimed to assist physicians in identifying patients at a high pLOS risk and thus to provide early and timely interventions for these high-risk patients.

Predictor variables were determined on the basis of prior studies $[23,24,28,29]$ and variable availability on admission. Variables used as predictor variables for model development in this study included age, sex, nationality, reason for admission, specific causes of chronic kidney disease (CKD), comorbidities, admission type, number of hospitalizations within 6 months, number of emergency admissions within 6 months, admission department, planned admission or not, admission day of the week, admitted in the same hospital as last admission or not, place of residence, and insurance type. The reason for admission, specific causes of CKD, and comorbidities were extracted using ICD-10 codes. The categories of reasons for admission and comorbidities were determined after consultation with experienced clinicians. Limited by the available data set, the number of hospitalizations within 6 months and number of emergency admissions within 6 months were calculated on the basis of the data collected from Class 3 hospitals.

\section{Model Development}

\section{RF Model}

$\mathrm{RF}$ is a supervised ensemble learning algorithm consisting of a collection of tree-structured classifiers [47]. RF models work by generating a multitude of decision trees independently and then synthesizing the individual predictions of all trees through a voting system. Each tree in an RF model is built using a bootstrap sample of the training data set. Assuming that $M$ predictor variables are included for model development, $F$ of all $M$ input variables are randomly selected for each node, and the split of each node is performed according to the minimal impurity principle. For each tree, a variable that was used for tree growth in the previous nodes will no longer be used in later splitting. In decision tree induction, the Gini index is a general impurity measure used to determine the splitting variables. If a data set $D$ contains samples with $J$ classes, the Gini index of data set $D-\operatorname{Gini}(D)$ — is defined as follows [48]:

$$
\operatorname{Gini}(D)=1-\sum_{j=1}^{J} p_{j}^{2}
$$

where $p_{j}$ is the frequency of the $j$ th class in $D$. At each node, if a variable can split the parent data set $D$ into 2 child data sets, $D_{1}$ and $D_{2}$, the decrease in the Gini index, $S$, for this variable is defined by the following:

$$
S=\operatorname{Gini}(D)-\operatorname{Gini}\left(D_{1}\right)-\operatorname{Gini}\left(D_{2}\right)
$$

The variable with a maximal decrease in the Gini index will be used for splitting at this node.

In an RF model, to classify a new case, each tree in the forest model gives a classification result for the new case as a vote, and the majority vote is declared as the final classification of the model. Twice randomization in an RF model, which involves randomly selecting training data samples and randomly selecting the attributes for each tree growth, provides the model with a strong capability of handling high-dimensional data together with a stable generalization error [49].

We used the RandomForestClassifier package in Python to construct the RF model in this study. A set of optimal parameters of the RF model was found using grid search, which is an exhaustive searching method using a manually specified subset of hyperparameter space to find the optimal parameters of a learning algorithm [50]. The RF model obtained in this study had the following parameters: the number of decision trees was 300 , the number of variables $(F)$ selected at each node was 10 , and the maximal depth of each decision tree was 28 .

\section{SVM Model}

SVMs have been used frequently in various classification problems because of their remarkably robust performance in handling noisy and nonlinearly classified data [51]. If the data set is not linearly separable, a mapping function will be used in the SVM to map the data set into a high-dimensional space. An SVM tries to find an optimal separating hyperplane (ie, the maximum-margin hyperplane) in the high-dimensional space to make a classification. Assuming that a training data set, $D$, consists of $N$ labeled cases, $D=\left\{\left(x_{1}, \mathrm{y}_{1}\right), \ldots,\left(x_{i}, y_{i}\right), \ldots,\left(x_{N}, y_{N}\right)\right\}$, where $x_{i}$ represents the $i$ th feature vector and $y_{i}$ is the label of the $i$ th case. A mapping function, $\varnothing(x)$, will map the data set from the original space into a high-dimensional space. In the transformed high-dimensional space, the separating hyperplane [52] is defined as follows:

$$
w^{\mathbf{T}} \phi(x)+b=0
$$

where is a normal vector determining the direction, and $b$ is the bias. The training cases with minimum margins from the hyperplane are called support vectors. A support vector $\left(x_{j}, y_{j}\right)$ satisfies:

$$
\left|w^{\mathrm{T}} \phi(x)+b\right|=1
$$

In the high-dimensional space, the margin $M$ between the support vector and the hyperplane is defined as

$$
M=\frac{1}{\|w\|}
$$

The hyperplane that makes the margin $M$ maximum is the optimal separating hyperplane (ie, maximum-margin hyperplane). In the process of finding the optimal separating hyperplane, a kernel function is usually used to deal with the high computational cost. Commonly used kernel functions include the polynomial kernel, the linear kernel, the exponential kernel, and the radial basis function kernel.

We used the svm package in Python to construct the SVM model, and the optimal parameters of our SVM model were found using grid search. The SVM model obtained in this study had the following parameters: the kernel function was polynomial kernel, the degree of the polynomial kernel function was 2 , and the penalty parameter $\mathrm{C}$ was 0.01 . 


\section{KNN Model}

KNN is a type of instance-based learning method that makes predictions based on a small number of cases that are very similar to the target observation [53]. Specifically, given a new case $\left(x_{\text {new }}\right)$, we can find the $\mathrm{K}$ closest training cases, $x_{(i)}(i=1, \ldots, K)$, sorted by the distance to $x_{\text {new }}$, and then classify $x_{\text {new }}$ using majority voting among the K neighbors. A commonly used distance metric in the KNN algorithm is the Euclidean distance. Assuming the presence of case $x_{i}=\left(p_{1}, p_{2}, \ldots, p_{M}\right)$ and $x_{j}=\left(q_{1}, q_{2}, \ldots, q_{M}\right)$, we define the Euclidean distance from $x_{i}$ to $x_{j}$ as

$$
d\left(x_{i}, x_{j}\right)=\sqrt{\left(p_{1}-q_{1}\right)^{2}+\left(p_{2}-q_{2}\right)^{2}+\cdots+\left(p_{M}-q_{M}\right)^{2}}
$$

where $p_{1}, p_{2}, \ldots, p_{M}$ and $q_{1}, q_{2}, \ldots, q_{M}$ denote the values of $M$ input predictor variables of the 2 cases. Typically, we first normalize all the values of the variables to the range of $(0,1)$ because different variables could be measured in different units. The KNN algorithm yields convincing results in handling various classification problems in medicine [54-56]. The model is effective on data sets where samples of 1 class have many possible patterns and the decision boundary is nonlinear [57]. The most important parameter in the KNN model is the number of neighbors, which must be selected with care. In this study, we used the KNeighborsClassifier package in Python to construct the KNN model. The optimal parameter $K$ was found using grid search, and the $\mathrm{KNN}$ model with optimal performance was obtained with the parameter $K=130$.

\section{Stacked Generalization}

Stacked generalization, or stacking, is an ensemble model that can combine the predictions of several primary machine learning models [37]. There are 2 types of models in a stacking framework: several base models (level-0 models) and 1 meta-model (level-1 model). The meta-model is employed to combine the base models. In general, a stacking framework can obtain a more accurate prediction result than any single base model. Different models may complement each other, and the meta-algorithm can combine the advantages of these base models.

The stacking model is trained as follows. Given a data set $D=\left\{\left(x_{1}, \mathrm{y}_{1}\right), \ldots,\left(x_{i}, y_{i}\right), \ldots,\left(x_{N}, y_{N}\right)\right\}$, we define $D_{k}$ and $D_{-k}=D-D_{k}$ as the training and test data sets, respectively, in the $k$ th round of model training. We assume that the stacking model has $J$ base models $\left(\right.$ Model $_{1}, \operatorname{Model}_{2}, \ldots, M_{\text {odel }}, \ldots$ Model $\left._{J}\right)$ and that each base model is trained using $D_{k}$. Let $\hat{y}_{j i}$ denote the prediction outcome produced by Model $_{j}$ for training case $\left(x_{i}, y_{i}\right)$. The outputs of all $J$ base models are assembled as the input of the meta-model. Let $\hat{Y}_{i}=\left(\hat{y}_{1 i}, \hat{y}_{2 i}, \ldots, \hat{y}_{j i}, \ldots, \hat{y}_{j i}\right)$ denote the set of outputs produced by all of the $J$ base models for $\left(x_{i}, y_{i}\right)$. The meta-model is then trained using data set $D_{\text {meta }}=\left\{\left(\hat{Y}_{1}, y_{1}\right), \ldots,\left(\hat{Y}_{i}, y_{i}\right), \ldots,\left(\hat{Y}_{N}, y_{N}\right)\right\}$.

For a new input case, the output of the meta-model is the final prediction outcome produced by the stacking model for the case. How the base models are assembled in the stacking method and how the prediction outcome for a new input case is generated by the stacking model are shown in Figure 1.

Figure 1. Stacked generalization, where Predictionj denotes the prediction outcome produced by the model (Modelj) for a new case.

\section{Base models}

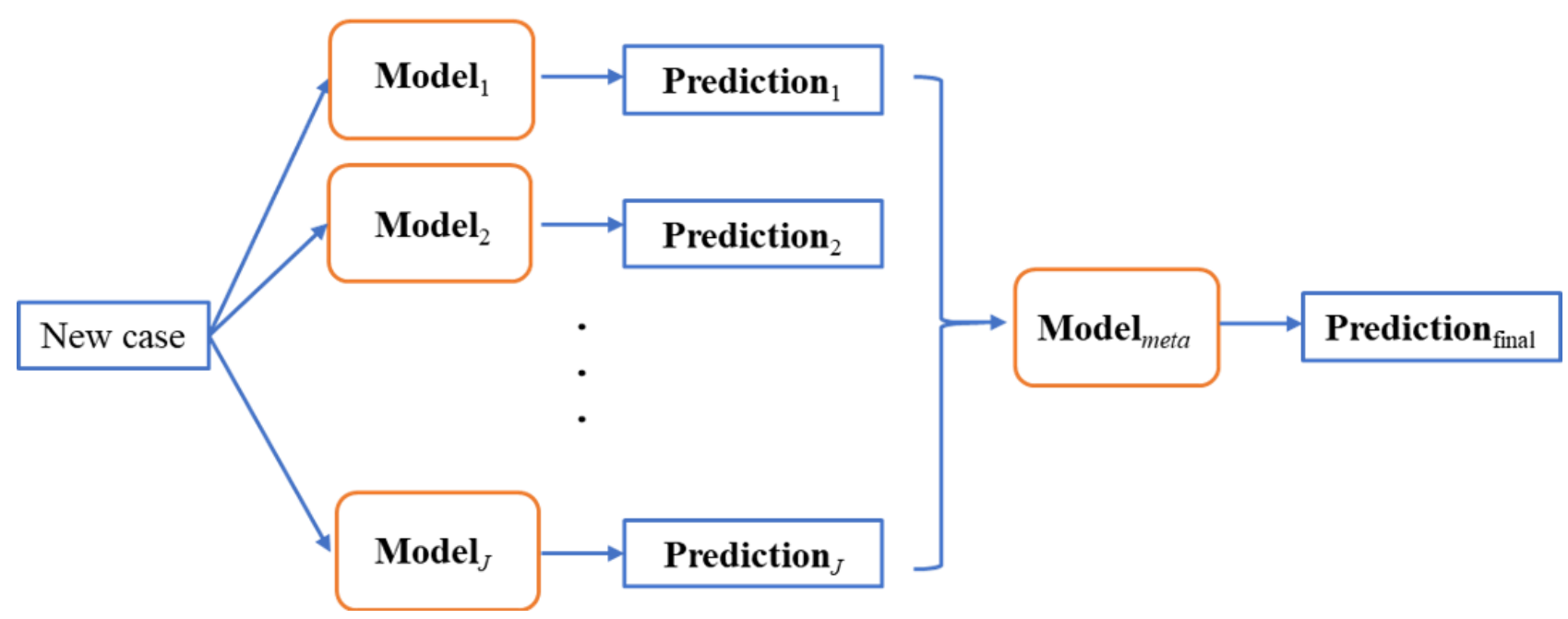

Given that the level-0 base models have already completed most of the prediction work, the level-1 meta-model could be rather simple [58]. The LR model is commonly used as the meta-model. Existing studies [37,59] suggested that increasing diversity of the base models could help improve the performance of the stacking model. In this study, the RF, SVM, and KNN models were employed as the base models and the LR model was used as the meta-model.

\section{Statistical Analysis}

Two-sided $t$ tests and chi-square tests were used for comparisons of patient demographics. In model development and comparisons, we employed 5-fold cross-validation. In 
performance comparisons, the Brier score [60], area under the receiver operating characteristic curve (AUROC) [60], estimated calibration index (ECI) [61], accuracy, sensitivity, specificity, and geometric mean $(\mathrm{Gm})$ [62] were employed as performance measures. Considering that other performance metrics, such as positive and negative predictive values and likelihood ratios, can be calculated from sensitivity and specificity, we did not employ them in performance comparisons. Brier score is an overall performance measure, with a lower Brier score suggesting a superior overall prediction performance. AUROC measures the discrimination power of a prediction model, representing the ability to distinguish positive samples from negative samples. ECI measures the calibration power of a model, representing the average difference between the predicted probabilities of individual patients and the observed probability in that patient population. ECI ranges between 0 and 100, with a lower ECI suggesting a stronger calibration power of the corresponding model. Gm is considered a balanced accuracy measure because it incorporates sensitivity and specificity, and it is defined as follows:

$$
\mathrm{Gm}=\sqrt{\text { Sensitivity } \times \text { Specificity }}
$$

Gm measures the balance of the classification performance for the majority and minority classes. The optimal cutoff value for each model was obtained according to its corresponding receiver operating characteristic curve, and then accuracy, sensitivity, specificity, and Gm were calculated. Performance differences between different models were assessed using 2-sided $t$ tests.
Furthermore, we used the calibration plot [60] to demonstrate the calibration power of each model in different patient groups with pLOS risk from low to high. In the calibration plot, patients were divided into 10 groups according to their predicted pLOS probabilities. The $\mathrm{x}$-axis shows the observed pLOS probability of each patient group, and the y-axis shows the averaged predicted pLOS probability of each group. The ideal calibration curve for a perfect model is a diagonal, which suggests that the predicted probabilities are exactly consistent with the observed probabilities.

Statistical analysis and calculations were performed using Python 3. Less than $15 \%$ of records in the HQMS database had missing values for the nationality and admission type variables, and the missing values were considered as a special category in the analysis.

\section{Results}

A total of 23,992 eligible patients receiving PD were included in our study, of whom $30.3 \%$ had a pLOS. Characteristics of the PD-treated patients are displayed in Table 1. The proportion of male patients was $55.6 \%(13,351 / 23,992)$, and the average age of all patients was 52.1 (SD 15.0) years. The 2-sided $t$ tests showed that the differences in age, place of residence, and insurance type between PD-treated patients with a pLOS and those without a pLOS were statistically significant. The histogram of the LOS distribution of the PD-treated patients is displayed in Figure 2. 
Table 1. Characteristics of peritoneal dialysis-treated patients in the study.

\begin{tabular}{|c|c|c|c|c|}
\hline Characteristic & All patients & Patients with $\mathrm{pLOS}^{\mathrm{a}}$ & Patients without pLOS & $P$ value \\
\hline Number of patients (\%) & $23,992(100)$ & $7270(30.3)$ & $16,722(69.7)$ & \\
\hline Age (years), mean (SD) & $52.1(15.0)$ & $53.6(15.4)$ & $51.5(14.8)$ & $<.001$ \\
\hline Sex, n (\%) & & & & .63 \\
\hline Female & $10,641(44.4)$ & $3242(44.6)$ & $7399(44.2)$ & \\
\hline Male & $13,351(55.6)$ & $4028(55.4)$ & $9323(55.8)$ & \\
\hline Place of residence, $n(\%)$ & & & & $<.001$ \\
\hline East China & $9425(39.3)$ & $2565(35.3)$ & $6860(41.0)$ & \\
\hline North China & $2318(9.7)$ & $902(12.4)$ & $1416(8.5)$ & \\
\hline Central China & $3416(14.2)$ & 1157 (15.9) & $2259(13.5)$ & \\
\hline South China & 3978 (16.6) & $1261(17.3)$ & $2717(16.2)$ & \\
\hline Southwest China & $2933(12.2)$ & 849 (11.7) & $2084(12.5)$ & \\
\hline Northwest China & $1067(4.4)$ & $225(3.1)$ & $842(5.0)$ & \\
\hline Northeast China & $855(3.6)$ & $311(4.3)$ & $544(3.3)$ & \\
\hline Insurance, $\mathrm{n}(\%)$ & & & & .005 \\
\hline UEBMI $^{\mathrm{b}}$ & $9100(37.9)$ & $2714(37.3)$ & $6386(38.2)$ & \\
\hline $\mathrm{URBMI}^{\mathrm{c}}$ & $2192(9.1)$ & $705(9.7)$ & $1487(8.9)$ & \\
\hline $\mathrm{NRCMS}^{\mathrm{d}}$ & $6082(25.4)$ & $1931(26.6)$ & $4151(24.8)$ & \\
\hline Free medical care & $334(1.4)$ & $101(1.4)$ & $233(1.4)$ & \\
\hline Self-paid treatment & 3493 (14.6) & $997(13.7)$ & $2496(14.9)$ & \\
\hline Other & 2791 (11.6) & $822(11.3)$ & 1969 (11.8) & \\
\hline
\end{tabular}

${ }^{a}$ pLOS: prolonged length of stay.

${ }^{\mathrm{b}}$ UEBMI: urban employee basic medical insurance.

${ }^{\mathrm{c}}$ URBMI: urban resident basic medical insurance.

${ }^{\mathrm{d}}$ NRCMS: new rural cooperative medical system.

Figure 2. Histogram of length of stay (LOS) distribution of peritoneal dialysis-treated patients.

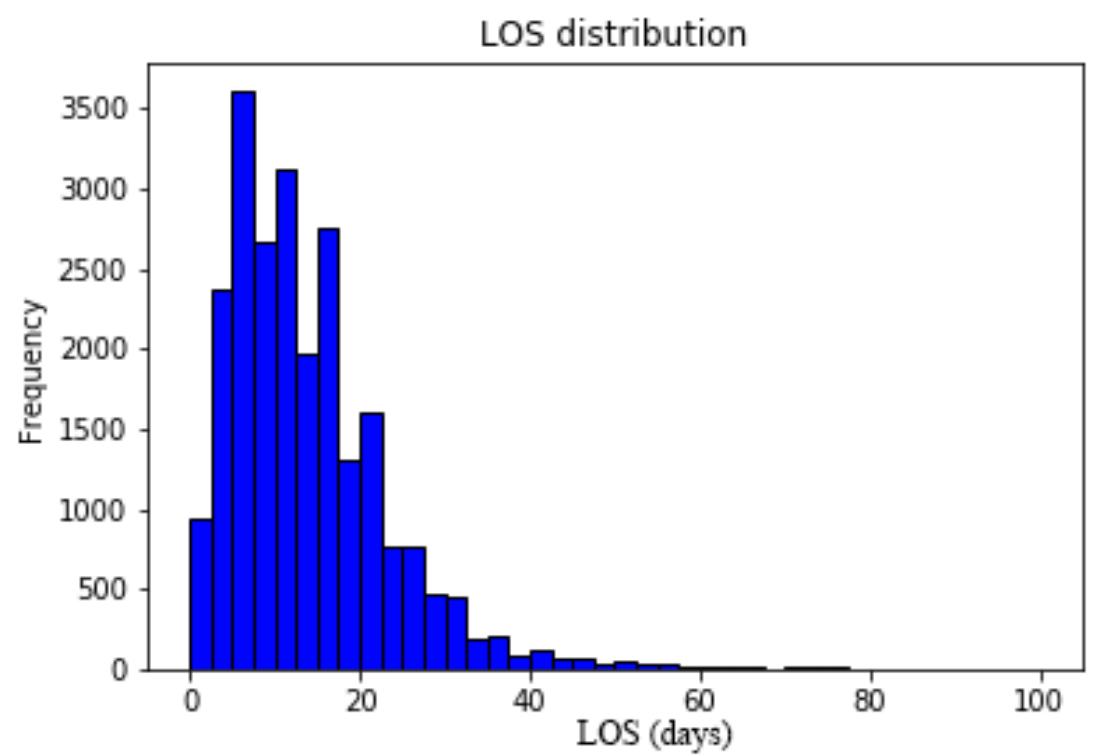

A comparison of the prediction performance of the stacking model, its 3 base models, and the benchmark LR model in terms of the Brier score, AUROC, ECI, Gm, accuracy, sensitivity, and specificity is shown in Table 2. Among these models, the 
stacking model achieved the best calibration (ECI 8.691), balanced accuracy (Gm 0.690), accuracy (0.695), and specificity (0.701). Meanwhile, the stacking and RF models had the best overall performance (Brier score 0.174 for both) and discrimination (AUROC 0.757 for the stacking model and 0.756 for the RF model). Compared with the benchmark LR model, the stacking model was superior in all performance measures except sensitivity, but there was no significant difference in sensitivity between the 2 models. The 2 -sided $t$ tests revealed significant performance differences between the stacking and LR models in overall performance, discrimination, calibration, balanced accuracy, and accuracy.

Table 2. Prediction performance of the 5 models.

\begin{tabular}{llllllll}
\hline Model & Brier score & AUROC $^{\mathrm{a}}(95 \% \mathrm{CI})$ & $\mathrm{ECI}^{\mathrm{b}}$ & $\mathrm{Gm}^{\mathrm{c}}$ & Accuracy & Sensitivity & Specificity \\
\hline $\mathrm{LR}^{\mathrm{d}}$ & 0.178 & $0.742(0.731-0.753)$ & 8.911 & 0.677 & 0.675 & 0.683 & 0.671 \\
$\mathrm{KNN}^{\mathrm{e}}$ & $0.188^{*}$ & $0.721(0.703-0.740)^{*}$ & $9.386^{*}$ & $0.661^{*}$ & 0.666 & 0.666 & 0.657 \\
$\mathrm{SVM}^{\mathrm{f}}$ & $0.187^{*}$ & $0.730(0.720-0.739)^{*}$ & $9.342^{*}$ & 0.673 & 0.680 & 0.656 & 0.690 \\
$\mathrm{RF}^{\mathrm{g}}$ & $0.174^{*}$ & $0.756(0.748-0.765)^{*}$ & $8.722^{*}$ & $0.689^{*}$ & $0.691^{*}$ & 0.686 & 0.693 \\
Stacking & $0.174^{*}$ & $0.757(0.748-0.765)^{*}$ & $8.691^{*}$ & $0.690^{*}$ & $0.695^{*}$ & 0.680 & 0.701 \\
\hline
\end{tabular}

${ }^{a}$ AUROC: area under the receiver operating characteristic curve.

${ }^{\mathrm{b}} \mathrm{ECI}$ : estimated calibration index.

${ }^{\mathrm{c}} \mathrm{Gm}$ : geometric mean.

${ }^{\mathrm{d}} \mathrm{LR}$ : logistic regression.

${ }^{\mathrm{e}} \mathrm{KNN}$ : K-nearest neighbor.

${ }^{\text {f }}$ SVM: support vector machine.

${ }^{\mathrm{g}} \mathrm{RF}$ : random forest.

$* P<.05$ in 2 -sided $t$ test when compared with the LR model.

Figure 3 demonstrates the calibration plots of the 5 models. The calibration curve of the stacking model was the optimal fitting curve among the 5 models. The SVM model underestimated the pLOS probabilities for most patients, whereas the KNN model overestimated the pLOS probabilities for most patients. The RF model underestimated the pLOS probabilities for most patients at low risk and overestimated the probabilities for most patients at high risk. 
Figure 3. Calibration plots of the 5 models. KNN: K-nearest neighbor; LR: logistic regression; RF: random forest; SVM: support vector machine.
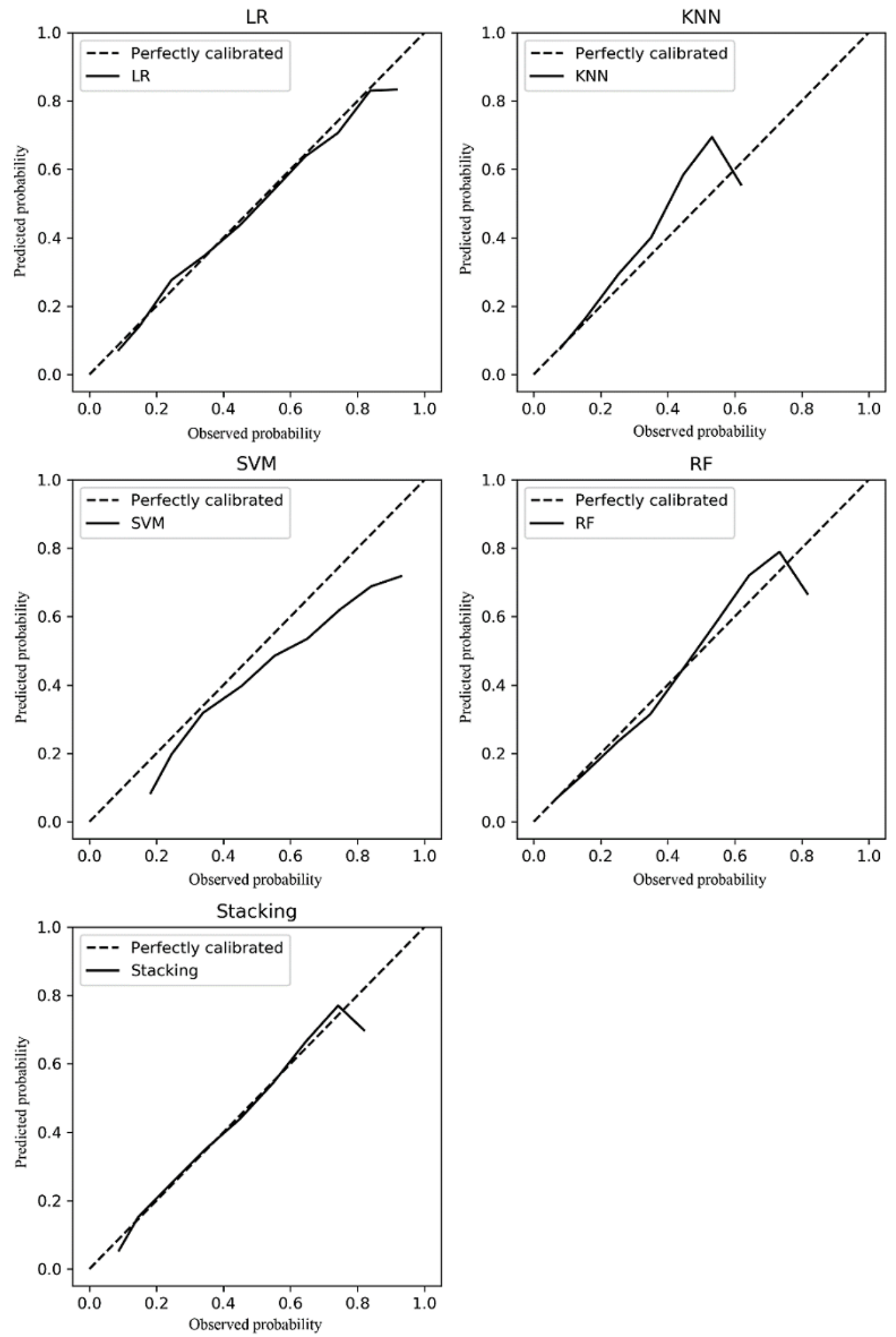

\section{Discussion}

\section{Principal Findings}

The main objective of this study was to develop an optimal data-driven model for predicting the pLOS risk of PD-treated patients using basic admission data. To the best of our knowledge, this study is the first to develop such pLOS prediction models for PD-treated patients by using data from a

national database. Our study constructed a pLOS prediction model for PD-treated patients based on a stacking method with $\mathrm{KNN}$, SVM, and RF as its base models and LR as its meta-model. The prediction performance of the stacking model was compared with those of a benchmark LR model and its 3 base models. A pragmatic pLOS prediction model for PD-treated patients would be useful in family consultation and has the potential to assist physicians in making optimal clinical decisions. Considering that medical expenses are highly 
associated with LOS [6,7], the pLOS prediction model could help estimate the medical expenses for PD-treated patients. The degree of satisfaction may increase if patients and their families know more about their LOS and medical expenses on hospital admission. In addition, the pLOS prediction models could be integrated into hospital information systems, providing physicians with real-time suggestions about the LOS of patients and helping physicians to identify PD-treated patients at a high risk of pLOS and give timely individualized intervention.

In this study, the RF, SVM, and KNN models were employed as base models for stacking because they have different learning mechanisms and have advantages in different aspects. RF is an ensemble learning algorithm consisting of a collection of tree-structured classifiers. The twice randomization in an RF model provides the model with a strong capability of handling high-dimensional data together with a stable generalizability [49]. However, RF models are sensitive to noise data. SVM models make classifications by mapping data into a high-dimensional space and finding an optimal separating hyperplane in the high-dimensional space. SVM models show remarkably robust performance in handling noisy and nonlinearly classified data but have limitations in handling high-dimensional data [51]. KNN is an instance-based learning method that makes predictions depending on a small number of cases that are strongly similar to the target observation. KNNs are effective on nonlinearly separable data sets and data sets where samples of one class have different patterns [57]. KNNs are insensitive to noise data but have limited accuracy in unbalanced data. In addition, an existing study [38] showed that the ensemble of the 3 models demonstrated superior prediction performance in dealing with classification problems. Moreover, the literature states that the 3 classifiers are suitable for pLOS prediction problems. All 3 classifiers have shown superior performance in predicting pLOS for patients. Chuang et al [35] employed the SVM and RF models for pLOS prediction in patients who underwent general surgery, and both models achieved a high AUROC. Steele and Thompson [63] developed a KNN-based pLOS prediction model for general patients and achieved an AUROC of 0.847 . KNN was included as the base model in our study because it has shown superior performance in pLOS prediction in existing studies [63,64]. Given that its learning mechanism is different from the learning mechanisms of the 2 other base models (SVM and RF), KNN was expected to improve the prediction performance of the stacking model in dealing with data sets with various characteristics [37,59]. We also attempted to construct stacking models with combinations of any 2 base models of RF, SVM, and KNN. We found that the stacking model with SVM and KNN as its base models had the worst performance, while the stacking model with 3 base models and the stacking models with the other 2 combinations (SVM and RF, and KNN and RF) had similar overall performances. Considering the diversity and respective advantages of the base models, and the generalizability of the stacking model in dealing with data sets with different characteristics, we selected the stacking model with 3 base models.

The performance comparison results showed that the stacking model was the best among the 5 models in terms of overall performance (Brier score), discrimination (AUROC), calibration (ECI), balanced accuracy (Gm), accuracy, and specificity. The RF model showed the best prediction performance among the 3 base models, and it had a similar overall performance and discrimination power as the stacking model. The good prediction performance of the stacking and RF models may be due to the fact that both models are ensemble learning models. Our study results are consistent with previous studies showing that the ensemble model is almost always superior to single learning models [38,39]. A stacking model can exploit its base models by combining the output of each model via a meta-model, thus reducing the bias that tends to occur with a single classifier. An RF model can exploit its base tree models by combining the output of each model via a voting system. The stacking model was slightly superior to the RF model in most performance measures for 2 possible reasons. First, the prediction performance of a stacking model is usually similar to its best base model [40,41]. Second, compared with an RF model, a stacking model has more diverse base models that can complement each other.

The calibration curves of the 5 models further suggest that the stacking model had the optimal calibration power in different patient groups. ECI measures the overall calibration power of a model, whereas the calibration curve visually shows the calibration power of a model in patient groups with pLOS risk from low to high. The ECI and calibration curve demonstrated that the stacking model had superior calibration power. The calibration curve showed that the averaged predicted pLOS probability of the stacking model had high consistency with the observed outcome across different pLOS risk groups. Meanwhile, the calibration curve showed that the RF model underestimated the pLOS probabilities of most patients at low risk and overestimated the probabilities of most patients at high risk. This feature can help the RF model expand the difference of predicted probabilities between patients with different pLOS risks and thus discriminate the patients at a high pLOS risk from those at a low risk. This probably explained why the RF model showed similar discrimination but worse calibration power than the stacking model.

We also attempted to develop numeric LOS prediction models for PD-treated patients, but the corresponding prediction performance of the models was limited, which was similar to that of existing numeric LOS prediction models. Numeric LOS prediction models focused on mining different LOS patterns for patients with different LOSs (even 1 day apart), but the difference in LOS patterns between patients with different LOSs, especially those LOSs with 1 or 2 days apart, may be slight and was difficult to identify. The pLOS prediction models with binary outcomes had a much better performance.

Regarding data exclusion, the PD-treated patients who died in the hospital were excluded in our study because the LOS pattern of the decedents might be different from that of patients who survived in the hospital $[65,66]$. Based on our consultations with experienced clinicians, we knew that there was uncertainty in the LOS pattern of patients who died in the hospital. Specifically, deceased patients could die quickly after hospital admission and have a short LOS or die after a long period of treatment and have a long LOS. In fact, the proportion of 
PD-treated patients who died in the hospital was only $0.8 \%$ in our study. Selection bias might have occurred when we excluded those PD-treated patients who died in the hospital, and the pLOS prediction model developed in our study may not apply to those patients who have a high risk of in-hospital mortality.

In our study, some PD-treated patients were hospitalized more than once; they can be classified into 2 types: (1) patients readmitted on the same day as discharge, and (2) patients with several discontinuous hospitalizations. Some hospitals in China may discharge patients with a potential pLOS first and then readmit them on the same day to reduce the average LOS, which is an important indicator in hospital evaluation. Therefore, for the PD-treated patients readmitted on the same day as discharge, we recalculated their actual LOS by merging the back-to-back admission records in this study. To deal with the situation of PD-treated patients with several discontinuous hospitalizations, we examined 2 approaches that were employed in the literature: (1) selecting the first hospitalization record, or (2) randomly selecting 1 record among multiple hospitalization records. Compared with the former approach, the latter approach may help include patients with varying severities [67]. Thus, we employed the second approach and randomly selected 1 record for each patient to ensure that all observations were independent and PD-treated patients with varying severities were included in model development.

\section{Definition of pLOS}

In this study, pLOS was defined as an LOS longer than the average LOS by referring to existing studies [44-46] and consulting with experienced clinicians. In the literature, there is no consensus on the definition of pLOS for general patients or PD-treated patients. Existing studies have defined pLOS as an LOS longer than the average LOS [44-46], longer than the median LOS [68], or longer than a specific LOS according to experiences [69]. After consulting with experienced clinicians, we know that the average LOS is a more important metric for PD-treated patients, and it is also a more commonly used metric in assessing medical efficiency around the world. In addition, pLOS has been defined as an LOS longer than the average LOS in various medical fields by researchers from different countries [44-46]. Among the 3 cited references that defined pLOS as an LOS longer than the average LOS, one study [44] was of trauma patients in the United States, another study [45] was of critically ill patients in Switzerland, and the third study [46] was of surgery patients in China. Therefore, the definition of pLOS as longer than the average LOS may help our models achieve good generalizability to some extent.

\section{Diagnosis Codes}

The use of diagnosis codes to identify patients with specific diseases may miss some target patients because clinicians tend to focus on the main diagnosis related to admission reasons and overlook the diagnosis of other diseases. To address this problem, we employed ICD-10 codes associated with all admission and discharge diagnoses and in-hospital medical operations to identify PD-treated patients. We also used ICD-10 codes associated with admission and discharge diagnoses to identify patients' comorbidities.

\section{Strengths and Limitations of the Study}

This study has several strengths. First, a large nationwide database with a relatively representative population was used to derive the prediction models. Second, all of the predictor variables are available at admission, which ensures the feasibility of applying the developed models in clinical practice to assist clinical decision making. Third, 5-fold cross-validation was employed to achieve reliable performance results.

However, this study has some limitations. First, the models were derived from a nationwide data set in China. Some of the variables included in the models, such as nationality and insurance type, are region specific. The generalizability and validity of our prediction models need to be validated using a data set from different regions. Second, other potentially important variables, such as some laboratory markers, that reportedly affect LOS $[27,70]$ were not available in the studied data set. Third, only patient data from Class 3 hospitals were included in the studied data set. Class 3 hospitals in China provide the best medical services for patients, and patients admitted to Class 3 hospitals in China may be suffering from serious diseases. Thus, our pLOS prediction models may not be applicable to the PD-treated patients in the primary or Class 2 hospitals in China, considering that patients admitted to those hospitals may have only minor or moderate diseases.

\section{Conclusion}

This study was the first to develop data-driven automated pLOS prediction models for PD-treated patients using basic admission data from a national database. The results of our study indicate the feasibility of utilizing a stacking-based model for PD-treated patients. The developed pLOS prediction models have the potential to help clinicians identify PD-treated patients at a high risk of pLOS and then provide optimal patient management. The pLOS prediction tools developed in this study have the potential to assist clinicians in identifying patients at a high risk of pLOS and to allocate resources optimally for PD-treated patients. The generalizability and validity of the developed pLOS prediction models need to be externally validated, and the clinical utility of the models needs further validation before they are used in clinical practice. The pLOS prediction models developed in our study are purely theoretical so far, and we plan to integrate them into the information system of a pilot hospital for prospective validation.

\section{Acknowledgments}

The authors thank the Bureau of Medical Administration and Medical Service Supervision, National Health Commission of China for the support of this study.

This study was supported by grants from the National Natural Science Foundation of China (Grant Nos. 81771938, 91846101, 82003529), Beijing Municipal Science \& Technology Commission (Grant No. 7212201), Beijing Nova Programme Interdisciplinary 
Cooperation Project (Z191100001119008), Chinese Scientific and Technical Innovation Project 2030 (2018AAA0102100), the University of Michigan Health System-Peking University Health Science Center Joint Institute for Translational and Clinical Research (BMU2020JI011, BMU2018JI012, BMU2019JI005), and PKU-Baidu Fund (2019BD017).

\section{Authors' Contributions}

Research idea and study design: GK, JW, LZ; data acquisition and preprocessing: YS, HW; data analysis and statistical analysis: JW, YL, CY, KL; the methodology for extracting patients and identifying disease: HC; supervision or mentorship: GK, LZ; manuscript writing: GK, JW, LZ. Each author contributed important intellectual content during manuscript drafting and accepts accountability for the overall work by ensuring that questions pertaining to the accuracy or integrity of any portion of the work are appropriately investigated and resolved.

\section{Conflicts of Interest}

None declared.

\section{Multimedia Appendix 1}

International Statistical Classification of Diseases, Tenth Revision (ICD-10) codes for identifying peritoneal dialysis patients. [DOCX File, 16 KB-Multimedia Appendix 1]

\section{References}

1. Saran R, Robinson B, Abbott KC, Agodoa LYC, Bragg-Gresham J, Balkrishnan R, et al. US Renal Data System 2018 Annual Data Report: Epidemiology of Kidney Disease in the United States. Am J Kidney Dis 2019 Mar;73(3 Suppl 1):A7-A8 [FREE Full text] [doi: 10.1053/j.ajkd.2019.01.001] [Medline: 30798791]

2. Liyanage T, Ninomiya T, Jha V, Neal B, Patrice HM, Okpechi I, et al. Worldwide access to treatment for end-stage kidney disease: a systematic review. Lancet 2015 May 16;385(9981):1975-1982. [doi: 10.1016/S0140-6736(14)61601-9] [Medline: 25777665]

3. Zhang L, Zuo L. Current burden of end-stage kidney disease and its future trend in China. Clin Nephrol 2016;86 (2016)(13):27-28. [doi: 10.5414/CNP86S104] [Medline: 27469147]

4. Zhang L, Zhao M, Zuo L, Wang Y, Yu F, Zhang H, CK-NET Work Group. China Kidney Disease Network (CK-NET) 2015 Annual Data Report. Kidney Int Suppl (2011) 2019 Mar;9(1):e1-e81 [FREE Full text] [doi: 10.1016/j.kisu.2018.11.001] [Medline: 30828481]

5. Chan KE, Lazarus JM, Wingard RL, Hakim RM. Association between repeat hospitalization and early intervention in dialysis patients following hospital discharge. Kidney Int 2009 Aug;76(3):331-341 [FREE Full text] [doi: 10.1038/ki.2009.199] [Medline: 19516243]

6. Higgins TL, McGee WT, Steingrub JS, Rapoport J, Lemeshow S, Teres D. Early indicators of prolonged intensive care unit stay: impact of illness severity, physician staffing, and pre-intensive care unit length of stay. Crit Care Med 2003 Jan;31(1):45-51. [doi: 10.1097/00003246-200301000-00007] [Medline: 12544992]

7. Lorentz CA, Leung AK, DeRosa AB, Perez SD, Johnson TV, Sweeney JF, et al. Predicting Length of Stay Following Radical Nephrectomy Using the National Surgical Quality Improvement Program Database. J Urol 2015 Oct;194(4):923-928. [doi: 10.1016/j.juro.2015.04.112] [Medline: 25986510]

8. Yu T, He Z, Zhou Q, Ma J, Wei L. Analysis of the factors influencing lung cancer hospitalization expenses using data mining. Thorac Cancer 2015 May;6(3):338-345 [FREE Full text] [doi: 10.1111/1759-7714.12147] [Medline: 26273381]

9. Lu M, Sajobi T, Lucyk K, Lorenzetti D, Quan H. Systematic review of risk adjustment models of hospital length of stay (LOS). Med Care 2015 Apr;53(4):355-365. [doi: 10.1097/MLR.0000000000000317] [Medline: 25769056]

10. Arora P, Kausz AT, Obrador GT, Ruthazer R, Khan S, Jenuleson CS, et al. Hospital utilization among chronic dialysis patients. J Am Soc Nephrol 2000 Apr;11(4):740-746 [FREE Full text] [Medline: 10752533]

11. Mathew AT, Strippoli GF, Ruospo M, Fishbane S. Reducing hospital readmissions in patients with end-stage kidney disease. Kidney Int 2015 Dec;88(6):1250-1260 [FREE Full text] [doi: 10.1038/ki.2015.307] [Medline: 26466320]

12. Bruns FJ, Seddon P, Saul M, Zeidel ML. The cost of caring for end-stage kidney disease patients: an analysis based on hospital financial transaction records. J Am Soc Nephrol 1998 May;9(5):884-890 [FREE Full text] [Medline: 9596087]

13. Chazan JA, London MR, Pono L. The impact of diagnosis-related groups on the cost of hospitalization for end-stage renal disease patients at Rhode Island Hospital from 1987 to 1990. Am J Kidney Dis 1992 Jun;19(6):523-525. [doi: 10.1016/s0272-6386(12)80829-8] [Medline: 1595699]

14. Goldstein SL, Smith CM, Currier H. Noninvasive interventions to decrease hospitalization and associated costs for pediatric patients receiving hemodialysis. J Am Soc Nephrol 2003 Aug;14(8):2127-2131 [FREE Full text] [doi: 10.1097/01.asn.0000076077.05508.7e] [Medline: 12874467] 
15. Li B, Cairns J, Fotheringham J, Ravanan R, ATTOM Study Group. Predicting hospital costs for patients receiving renal replacement therapy to inform an economic evaluation. Eur J Health Econ 2016 Jul;17(6):659-668. [doi: 10.1007/s10198-015-0705-x] [Medline: 26153418]

16. Menéndez R, Cremades M, Martínez-Moragón E, Soler J, Reyes S, Perpiñá M. Duration of length of stay in pneumonia: influence of clinical factors and hospital type. Eur Respir J 2003 Oct;22(4):643-648 [FREE Full text] [doi: 10.1183/09031936.03.00026103] [Medline: 14582918]

17. Abdelaziz TS, Fouda R, Hussin WM, Elyamny MS, Abdelhamid YM. Preventing acute kidney injury and improving outcome in critically ill patients utilizing risk prediction score (PRAIOC-RISKS) study. A prospective controlled trial of AKI prevention. J Nephrol 2020 Apr;33(2):325-334. [doi: 10.1007/s40620-019-00671-6] [Medline: $\underline{31712987]}$

18. Hachesu PR, Ahmadi M, Alizadeh S, Sadoughi F. Use of data mining techniques to determine and predict length of stay of cardiac patients. Healthc Inform Res 2013 Jun;19(2):121-129 [FREE Full text] [doi: 10.4258/hir.2013.19.2.121] [Medline: 23882417]

19. Douma CE, Redekop WK, van der Meulen JH, van Olden RW, Haeck J, Struijk DG, et al. Predicting mortality in intensive care patients with acute renal failure treated with dialysis. J Am Soc Nephrol 1997 Jan;8(1):111-117 [FREE Full text] [Medline: 9013455]

20. Wagner M, Ansell D, Kent DM, Griffith JL, Naimark D, Wanner C, et al. Predicting mortality in incident dialysis patients: an analysis of the United Kingdom Renal Registry. Am J Kidney Dis 2011 Jun;57(6):894-902 [FREE Full text] [doi: 10.1053/j.ajkd.2010.12.023] [Medline: 21489668]

21. Quinn R, Laupacis A, Hux J, Oliver M, Austin PC. Predicting the risk of 1-year mortality in incident dialysis patients: accounting for case-mix severity in studies using administrative data. Med Care 2011 Mar;49(3):257-266. [doi: 10.1097/MLR.0b013e318202aa0b] [Medline: 21301370]

22. Matsubara Y, Kimachi M, Fukuma S, Onishi Y, Fukuhara S. Development of a new risk model for predicting cardiovascular events among hemodialysis patients: Population-based hemodialysis patients from the Japan Dialysis Outcome and Practice Patterns Study (J-DOPPS). PLoS One 2017;12(3):e0173468 [FREE Full text] [doi: 10.1371/journal.pone.0173468] [Medline: 28273175]

23. Allon M, Radeva M, Bailey J, Beddhu S, Butterly D, Coyne D, HEMO Study Group. The spectrum of infection-related morbidity in hospitalized haemodialysis patients. Nephrol Dial Transplant 2005 Jun;20(6):1180-1186. [doi: 10.1093/ndt/gfh729] [Medline: 15769823]

24. Kshirsagar AV, Hogan SL, Mandelkehr L, Falk RJ. Length of stay and costs for hospitalized hemodialysis patients: nephrologists versus internists. J Am Soc Nephrol 2000 Aug;11(8):1526-1533 [FREE Full text] [Medline: 10906167]

25. Rocco MV, Soucie JM, Reboussin DM, McClellan WM. Risk factors for hospital utilization in chronic dialysis patients. Southeastern Kidney Council (Network 6). J Am Soc Nephrol 1996 Jun;7(6):889-896 [FREE Full text] [Medline: 8793798$]$

26. Fleischmann E, Teal N, Dudley J, May W, Bower JD, Salahudeen AK. Influence of excess weight on mortality and hospital stay in 1346 hemodialysis patients. Kidney Int 1999 Apr;55(4):1560-1567 [FREE Full text] [doi: 10.1046/j.1523-1755.1999.00389.x] [Medline: 10201023 ]

27. Ofsthun N, Labrecque J, Lacson E, Keen M, Lazarus JM. The effects of higher hemoglobin levels on mortality and hospitalization in hemodialysis patients. Kidney Int 2003 May;63(5):1908-1914 [FREE Full text] [doi: 10.1046/j.1523-1755.2003.00937.x] [Medline: 12675871]

28. Matas AJ, Gillingham KJ, Elick BA, Dunn DL, Gruessner RW, Payne WD, et al. Risk factors for prolonged hospitalization after kidney transplants. Clin Transplant 1997 Aug;11(4):259-264. [Medline: 9267712]

29. Viglino G, Cancarini G, Catizone L, Cocchi R, De Vecchi A, Lupo A, et al. Ten years experience of CAPD in diabetics: comparison of results with non-diabetics. Italian Cooperative Peritoneal Dialysis Study Group. Nephrol Dial Transplant 1994;9(10):1443-1448. [Medline: 7816258]

30. Moran JL, Solomon PJ, ANZICS Centre for OutcomeResource Evaluation (CORE) of the Australian New Zealand Intensive Care Society (ANZICS). A review of statistical estimators for risk-adjusted length of stay: analysis of the Australian and new Zealand Intensive Care Adult Patient Data-Base, 2008-2009. BMC Med Res Methodol 2012 May 16;12:68 [FREE Full text] [doi: 10.1186/1471-2288-12-68] [Medline: 22591115]

31. Yang C, Wei C, Yuan C, Schoung J. Predicting the length of hospital stay of burn patients: Comparisons of prediction accuracy among different clinical stages. Decis Support Syst 2010 Dec;50(1):325-335. [doi: 10.1016/j.dss.2010.09.001]

32. LaFaro RJ, Pothula S, Kubal KP, Inchiosa ME, Pothula VM, Yuan SC, et al. Neural Network Prediction of ICU Length of Stay Following Cardiac Surgery Based on Pre-Incision Variables. PLoS One 2015;10(12):e0145395 [FREE Full text] [doi: 10.1371/journal.pone.0145395] [Medline: 26710254]

33. Wolff J, McCrone P, Patel A, Kaier K, Normann C. Predictors of length of stay in psychiatry: analyses of electronic medical records. BMC Psychiatry 2015 Oct 07;15:238 [FREE Full text] [doi: 10.1186/s12888-015-0623-6] [Medline: 26446584]

34. Ma X, Si Y, Wang Z, Wang Y. Length of stay prediction for ICU patients using individualized single classification algorithm. Comput Methods Programs Biomed 2020 Apr;186:105224. [doi: 10.1016/j.cmpb.2019.105224] [Medline: 31765937]

35. Chuang M, Hu Y, Lo C. Predicting the prolonged length of stay of general surgery patients: a supervised learning approach. Int Tran Oper Res 2016 May 30;25(1):75-90. [doi: 10.1111/itor.12298] 
36. Morton A, Marzban E, Giannoulis G, Patel A, Aparasu R, Kakadiaris IA. A comparison of supervised machine learning techniques for predicting short-term in-hospital length of stay among diabetic patients. 2014 Presented at: International Conference on Machine Learning and Applications; Dec 3-6, 2014; Detroit, MI, USA p. 428-431. [doi: 10.1109/ICMLA.2014.76]

37. Wolpert DH. Stacked generalization. Neural Networks 1992 Jan;5(2):241-259. [doi: 10.1016/s0893-6080(05)80023-1]

38. Lertampaiporn S, Thammarongtham C, Nukoolkit C, Kaewkamnerdpong B, Ruengjitchatchawalya M. Heterogeneous ensemble approach with discriminative features and modified-SMOTEbagging for pre-miRNA classification. Nucleic Acids Res 2013 Jan 07;41(1):e21. [doi: 10.1093/nar/gks878] [Medline: 23012261]

39. Wang S, Yang J, Chou K. Using stacked generalization to predict membrane protein types based on pseudo-amino acid composition. J Theor Biol 2006 Oct 21;242(4):941-946. [doi: 10.1016/j.jtbi.2006.05.006] [Medline: 16806277]

40. Phan J, Hoffman R, Kothari S, Wu P, Wang MD. Integration of multi-modal biomedical data to predict cancer grade and patient survival. In: IEEE EMBS Int Conf Biomed Health Inform.: IEEE; 2016 Feb Presented at: 2016 IEEE-EMBS International Conference on Biomedical and Health Informatics (BHI); 24-27 Feb. 2016; Las Vegas, NV, USA p. $577-580$ URL: http://europepmc.org/abstract/MED/27493999 [doi: 10.1109/BHI.2016.7455963]

41. Pourhoseingholi M, Kheirian S, Zali M. Comparison of basic and ensemble data mining methods in predicting 5-year survival of colorectal cancer patients. Acta Inform Med 2017 Dec;25(4):254-258. [doi: 10.5455/aim.2017.25.254-258] [Medline: 29284916]

42. Zhang L, Wang H, Long J, Shi Y, Bai K, Jiang W, et al. China Kidney Disease Network (CK-NET) 2014 Annual Data Report. Am J Kidney Dis 2017 Jun;69(6S2):A4 [FREE Full text] [doi: 10.1053/j.ajkd.2016.06.011] [Medline: 28532549]

43. National Health Family Planning Commission. National Medical Service and Quality Safety Report. Beijing: People's Medical Publishing House; 2016.

44. Brasel KJ, Lim HJ, Nirula R, Weigelt JA. Length of stay: an appropriate quality measure? Arch Surg 2007 May;142(5):461-5; discussion 465. [doi: 10.1001/archsurg.142.5.461] [Medline: 17515488]

45. Zoller B, Spanaus K, Gerster R, Fasshauer M, Stehberger PA, Klinzing S, et al. ICG-liver test versus new biomarkers as prognostic markers for prolonged length of stay in critically ill patients - a prospective study of accuracy for prediction of length of stay in the ICU. Ann Intensive Care 2014;4:19 [FREE Full text] [doi: 10.1186/s13613-014-0019-7] [Medline: $\underline{25045579]}$

46. Song X, Xia C, Li Q, Yao C, Yao Y, Chen D, et al. Perioperative predictors of prolonged length of hospital stay following total knee arthroplasty: a retrospective study from a single center in China. BMC Musculoskelet Disord 2020 Jan 31;21(1):62 [FREE Full text] [doi: 10.1186/s12891-020-3042-x] [Medline: 32005208 ]

47. Breiman L. Random forests. Machine Learning 2001;45(1):5-32. [doi: 10.1023/A:1010933404324]

48. Kulkarni VY, Sinha PK, Petare MC. Weighted hybrid decision tree model for random forest classifier. J Inst Eng India Ser B 2015 Jan 3;97(2):209-217. [doi: 10.1007/s40031-014-0176-y]

49. Touw WG, Bayjanov JR, Overmars L, Backus L, Boekhorst J, Wels M, et al. Data mining in the life sciences with random forest: A walk in the park or lost in the jungle? Brief Bioinform 2013 May;14(3):315-326 [FREE Full text] [doi: 10.1093/bib/bbs034] [Medline: 22786785]

50. Hsu C-W, Chang C-C, Lin C-J. A practical guide to support vector classification. 2016. URL: https://www.csie.ntu.edu.tw/ cjlin/papers/guide/guide.pdf [accessed 2021-04-14]

51. Furey TS, Cristianini N, Duffy N, Bednarski DW, Schummer M, Haussler D. Support vector machine classification and validation of cancer tissue samples using microarray expression data. Bioinformatics 2000 Oct;16(10):906-914. [doi: 10.1093/bioinformatics/16.10.906] [Medline: 11120680]

52. Boser BE, Guyon IM, Vapnik VN. A training algorithm for optimal margin classifier. New York, NY, United States: Association for Computing Machinery; 1992 Presented at: COLT92: 5th Annual Workshop on Computational Learning Theory; July 1992; Pittsburgh, Pennsylvania p. 144-152. [doi: 10.1145/130385.130401]

53. Cover T, Hart P. Nearest neighbor pattern classification. IEEE Trans Inform Theory 1967 Jan;13(1):21-27. [doi: 10.1109/tit.1967.1053964]

54. Lu J, Getz G, Miska EA, Alvarez-Saavedra E, Lamb J, Peck D, et al. MicroRNA expression profiles classify human cancers. Nature 2005 Jun 09;435(7043):834-838. [doi: 10.1038/nature03702] [Medline: 15944708]

55. Sarasin-Filipowicz M, Oakeley EJ, Duong FHT, Christen V, Terracciano L, Filipowicz W, et al. Interferon signaling and treatment outcome in chronic hepatitis C. Proc Natl Acad Sci U S A 2008 May 13;105(19):7034-7039 [FREE Full text] [doi: 10.1073/pnas.0707882105] [Medline: 18467494]

56. Wang X, Yu J, Sreekumar A, Varambally S, Shen R, Giacherio D, et al. Autoantibody signatures in prostate cancer. N Engl J Med 2005 Sep 22;353(12):1224-1235. [doi: 10.1056/NEJMoa051931] [Medline: 16177248]

57. Hastie T, Tibshirani R, Friedman J. Prototype methods and nearest-neighbors. In: The Elements of Statistical Learning. New York, NY: Springer Science+Business Media; Jan 01, 2009:463-471.

58. Witten I, Frank E, Hall MA. Data Mining: Practical Machine Learning Tools and Techniques. San Francisco, CA: Morgan Kaufmann Publishers; Jan 01, 2011. 
59. Wang R. Significantly improving the prediction of molecular atomization energies by an ensemble of machine learning algorithms and rescanning input space: A stacked generalization approach. J Phys Chem C 2018 Apr 12;122(16):8868-8873. [doi: $10.1021 /$ acs.jpcc.8b03405]

60. Steyerberg E, Vickers A, Cook N, Gerds T, Gonen M, Obuchowski N, et al. Assessing the performance of prediction models: a framework for traditional and novel measures. Epidemiology 2010 Jan;21(1):128-138 [FREE Full text] [doi: 10.1097/EDE.0b013e3181c30fb2] [Medline: 20010215]

61. Van Hoorde K, Van Huffel S, Timmerman D, Bourne T, Van Calster B. A spline-based tool to assess and visualize the calibration of multiclass risk predictions. J Biomed Inform 2015 Apr;54:283-293 [FREE Full text] [doi: 10.1016/j.jbi.2014.12.016] [Medline: 25579635]

62. Hao M, Wang Y, Bryant SH. An efficient algorithm coupled with synthetic minority over-sampling technique to classify imbalanced PubChem BioAssay data. Anal Chim Acta 2014 Jan 02;806:117-127 [FREE Full text] [doi:

10.1016/j.aca.2013.10.050] [Medline: 24331047]

63. Steele R, Thompson B. Data mining for generalizable pre-admission prediction of elective length of stay. : IEEE; 2019 Presented at: IEEE 9th Annual Computing and Communication Workshop and Conference (CCWC); January 7-9, 2019; Las Vegas, NV p. 0127-0133. [doi: 10.1109/CCWC.2019.8666598]

64. Kumar A, Anjomshoa H. A two-stage model to predict surgical patients' lengths of stay from an electronic patient database. IEEE J Biomed Health Inform 2019 Mar;23(2):848-856. [doi: 10.1109/JBHI.2018.2819646] [Medline: 29993793]

65. Liew D, Liew D, Kennedy MP. Emergency department length of stay independently predicts excess inpatient length of stay. Med J Aust 2003 Nov 17;179(10):524-526. [doi: 10.5694/j.1326-5377.2003.tb05676.x] [Medline: 14609414]

66. Verburg IWM, de Keizer NF, de Jonge E, Peek N. Comparison of regression methods for modeling intensive care length of stay. PLoS One 2014;9(10):e109684 [FREE Full text] [doi: 10.1371/journal.pone.0109684] [Medline: 25360612]

67. Harel Z, Wald R, McArthur E, Chertow GM, Harel S, Gruneir A, et al. Rehospitalizations and emergency department visits after hospital discharge in patients receiving maintenance hemodialysis. J Am Soc Nephrol 2015 Dec;26(12):3141-3150 [FREE Full text] [doi: 10.1681/ASN.2014060614] [Medline: 25855772]

68. Aga Z, Machina M, McCluskey S. Greater intravenous fluid volumes are associated with prolonged recovery after colorectal surgery: A retrospective cohort study. Br J Anaesth 2016 Jun;116(6):804-810 [FREE Full text] [doi: 10.1093/bja/aew125] [Medline: 27199312]

69. Farjah F, Lou F, Rusch VW, Rizk NP. The quality metric prolonged length of stay misses clinically important adverse events. Ann Thorac Surg 2012 Sep;94(3):881-7; discussion 887. [doi: 10.1016/j.athoracsur.2012.04.082] [Medline: 22742847]

70. Noh H, Lee SW, Kang SW, Shin SK, Choi KH, Lee HY, et al. Serum C-reactive protein: a predictor of mortality in continuous ambulatory peritoneal dialysis patients. Perit Dial Int 1998;18(4):387-394. [Medline: 10505560]

\author{
Abbreviations \\ ANN: artificial neural network \\ AUROC: area under the receiver operating characteristic curve \\ CKD: chronic kidney disease \\ ECI: estimated calibration index \\ ESKD: end-stage kidney disease \\ Gm: geometric mean \\ HQMS: Hospital Quality Monitoring System \\ ICD-10: International Statistical Classification of Diseases, Tenth Revision \\ ICU: intensive care unit \\ KNN: K-nearest neighbor \\ LOS: length of stay \\ LR: logistic regression \\ PD: peritoneal dialysis \\ pLOS: prolonged length of stay \\ RF: random forest \\ SVM: support vector machine
}


Edited by C Lovis; submitted 22.01.20; peer-reviewed by YJ Tseng, A James, W Pian, S Sarbadhikari; comments to author 07.06.20; revised version received 10.08.20; accepted 07.03.21; published 19.05.21

Please cite as:

Kong G, Wu J, Chu H, Yang C, Lin Y, Lin K, Shi Y, Wang H, Zhang L

Predicting Prolonged Length of Hospital Stay for Peritoneal Dialysis-Treated Patients Using Stacked Generalization: Model Development and Validation Study

JMIR Med Inform 2021;9(5):e17886

URL: https://medinform.jmir.org/2021/5/e17886

doi: $\underline{10.2196 / 17886}$

PMID:

(C) Guilan Kong, Jingyi Wu, Hong Chu, Chao Yang, Yu Lin, Ke Lin, Ying Shi, Haibo Wang, Luxia Zhang. Originally published in JMIR Medical Informatics (https://medinform.jmir.org), 19.05.2021. This is an open-access article distributed under the terms of the Creative Commons Attribution License (https://creativecommons.org/licenses/by/4.0/), which permits unrestricted use, distribution, and reproduction in any medium, provided the original work, first published in JMIR Medical Informatics, is properly cited. The complete bibliographic information, a link to the original publication on https://medinform.jmir.org/, as well as this copyright and license information must be included. 